\title{
Elemental Design Base of Chinese Supercomputer Technologies
}

\author{
Andrey Molyakov ${ }^{1}$ \\ ${ }^{1}$ Institute of information technologies and cybersecurity, Russian State University for the Humanities, Moscow, \\ Russia \\ Correspondence: Andrey Molyakov, Institute of information technologies and cybersecurity, Russian State \\ University for the Humanities, Moscow, 117534, Kirovogradskaya street, 25/2, Russia.
}

Received: April 2, 2020

doi:10.5539/cis.v13n2p43
Accepted: April 23, 2020

Online Published: April 24, 2020

URL: https://doi.org/10.5539/cis.v13n2p43

\begin{abstract}
China intends to close the technological gap from Western countries in microprocessor production by 2021. 46 new projects worth billions of dollars have already been launched in the country. Most of these projects receive direct or indirect state support, which creates a huge demand for domestic semiconductors. By a government decree, all servers in government offices and state-owned enterprises will be preferentially equipped with processors of domestic production. As a result, their sales are expected to grow at a rate of $20 \%$ annually. First of all, we are talking about "Loongson", "Shenwei" and "Phytium" chips (the latter are the development of the Chinese military-industrial complex). The Shenwei processors, which have proved their effectiveness, are promising: they are the ones that run the world's most powerful supercomputer, Sunway Taihu Light, which consists of 10.65 million cores and performs 93 quadrillion operations per second.
\end{abstract}

Keywords: Sparc T-series, Tianhe, ICT, Godson-T, Project "Stork"

\section{Introduction}

The decision to switch to domestic processors was made by the state after, in the midst of U.S.- Chinese trade disputes, the United States imposed sanctions against the Chinese ZTE corporation in April, banning it from supplying microprocessors and other equipment. With the introduction of an embargo against ZTE, it became clear: in many Chinese computer and telecommunication equipment, the percentage of use of domestic microcircuits is extremely small. Experts called this a direct threat to national security. The Chinese reaction to this threat, in turn, will strike at Intel's largest US processor manufacturer, which will lose a huge market in the Chinese public sector. The volume of losses will be in the billions: today China annually imports $\$ 200$ billion worth of microprocessors (Eisymont \& Nikitin, 2016; Eisymont, 2016).

According to the elementary design base of China for high-performance computing systems and supercomputers, the following organizations and areas of work can be distinguished.

\section{National University of Defense Technology of China (NUDT), the Most Significant of the Known Developments}

Massive multi-threaded microprocessors (CT-2 / CT-3 projects) and 4D assembly technology with liquid direct cooling, hybrid heavy cores for China's global military intelligence information system (Sinyuan area). Microprocessors are developed on the basis of architectural principles embedded in the $\mathrm{J} 7 / \mathrm{J} 10$ microprocessors of the Russian Angara strategic supercomputer project, and the architecture of the system asa whole also uses the computational organization model of the American ParalleX project (Molyakov \& Eisymont, 2017).

Multi-threaded microprocessors FT-1000/1500/2000. These are modernized versions of the American SunNiagara-1, 2, and 3 microprocessors. Currently, these are Sparc T-series microprocessors.

Two types of chips for the Arch communication network with the bold tree topology used in the most powerful Chinese supercomputers - a chip for a network adapter and a multiport communication chip. It is known that during the development of these chips IBM materials were used on the PERCS network, and now they are preparing a deeply processed version of them, or even replacing them with the multipurpose HUB routers of the PERCS network used in IBM Power 775 supercomputers.

Tianhe-1 and Tianhe-2 supercomputers are used for military and civilian applications (Alba \& Davey, 2014). A modernized military version of Tianhe- 2 is being prepared, in which a new element base will be used in the 
communication network, as close as possible to the PERCS network. It is also possible to use the IBM Power 9 microprocessor in the future, an upgrade of which will be licensed at the TSMC factory according to Chinese technical proposals.

A sample of a 1024-qubit spin-quantum supercomputer was created using the technology of the Canadian company D-Wave. This work was carried out jointly with the University of Science and Technology of China (Hay-Wei Province). The application area of this supercomputer supposed by American analysts is cryptanalysis. Currently, 128- and 512-qubit versions of such supercomputers are available at Lockhead Martine (tasks of software verification of the F-35 fighter) and the NASA Evans Center (information systems (Google), robotics and UAV control systems (NASA). In the United States, 1024 and 2048 qubit variants of this type of supercomputer are being prepared for release in the next year or two. The interest in this type of supercomputer is caused by the fact that on some interesting problems its performance is 3-4 orders of magnitude higher than that of one standard modern superscalar microprocessor even in the 128-qubit version.

Research is being conducted on the elemental base of computers with reverse logic based on cellular automata and quantum dots. This in the long run will make it possible to overcome the physical performance constraint of Exaflops level associated with the Landauer thermodynamic constraint, but surmountable by virtue of the Neumann-Landauro principle, provided that reverse logic is used.

\section{Institute of Computer Technology, Chinese Academy of Sciences (ICT RAS)}

Godson (also well-known as Loongson) multi-core superscalar microprocessors with MIPS architecture, but supported by hardware-efficient emulation of the x86 architecture command system and reinforced by a powerful SIMD block for processing short vectors (Attig \& Norbert, 2012).

This is a type of microprocessors with "heavy" computing cores. Modern samples of interest: Goodson 3B - 65 $\mathrm{nm}$ technology, 8-core, peak performance 128 Gflops, 40 watts consumption; Godson 3C - $28 \mathrm{~nm}$ technology, 16-core, peak performance 384 GFlops, consumption of 20 watts (2012-2013); Godson 4A - 64-core (2012-2013); Godson 4B (2014-2015), microprocessor with many light Godson-T cores (64 cores), such as a Tilera microprocessor. Hybrid microprocessor, in the crystal of which Godson-3 and Godson-T will simultaneously be present.

SuperDragon-1 and -2 supercomputers for network intelligence systems with heavy hybrid nodes, which, for example, contain: 4 Loongson 3b processors, 2-4 NVIDIA Fermi or Kepler GPUs or up to four boards on each with 16 FPGA Xilinx Virtex-6 LX550T (GPU or FPGA boards are connected via 4 PCI-E 3.0 x16 slots).

The company SunWay, is supervised by the military-political administration, the development of computing tools of various capacities up to petaflops supercomputers, on its own element base in the form of microprocessors with the DEC Alpha architecture and Arch network for educational institutions, the economy and government institutions (CEA-HPC, 2012).

The creation of general-purpose supercomputers on the import and domestic element base for scientific and technical calculations and state structures of the highest echelon is carried out by the new Sugon integrator corporation, using developments from Dawning, Huawei, and ICT CAS. The most famous computing systems are Downing 4000, 5000, 6000 (today's time) and 7000 (the system of the near future). Less well known are systems with such numbers, but with letter indices at the end of A (advanced), L (light) and H (heavy). These indices indicate different options, from cheap and low-productivity, with imported components, to more expensive and productive, only on domestic components (Li Wei and others, 2016; Li Wei and others, 2017). The computing nodes of these supercomputers are essentially hybrid, for example, the Downing $6000 \mathrm{H}$ node contains: 4 Loongson 4A (each connected with links), 2 FT-2000 and 2-4 K20 GPUs (connected via PCI-E 3.0), Goodson-T can be used (Balfour and others, 2008; Khazraee Moeim and others, 2017; Eisymont \& Butov, 2016).

\section{Conclusion}

The main microelectronic factory in China is the Taiwanese TSMC, over $80 \%$ of the shares of which belong to mainland China, and whose deputy director is Colonel of Chinese military intelligence, Den Lu. One of his duties, in particular, is to conduct a line of embedding a "spyware" (NUDT project "Stork", development of hackers of the 15th zone) in microcircuits manufactured by this factory. IBM and Intel are actively participating in this factory, actively transferring the most advanced technologies in exchange for the possibility of using production capacities and pursuing a policy of China to block the transfer of high technologies and products to Russia, even to minimize existing contacts. For example, a line is being drawn up to curtail contacts with the Russian company Syntronics. 
Experts believe that TSMC will already surpass Intel factories on $14 \mathrm{~nm}$ technologies, and such strict adherence to the conditions for the transfer of high technologies from American companies can last until 2021, when China itself becomes quite independent in the field of high technologies. At the same time, China is cooperating with the European Union in the field of micro- and optoelectronics, modern constructs and electronic components of the Post-Moore's era.

\section{Acknowledgments}

Identify grants or other financial support (and the source, if appropriate) for your study; do not precede grant numbers by No. or \#. Next, acknowledge colleagues who assisted in conducting the study or critiquing the manuscript. Do not acknowledge the persons routinely involved in the review and acceptance of manuscripts peer reviewers or editors, associate editors, and consulting editors of the journal in which the article is to appear. In this paragraph, also explain any special agreements concerning authorship, such as if authors contributed equally to the study. End this paragraph with thanks for personal assistance, such as in manuscript preparation.

\section{References}

Alba, D. (2014, March 3). China's Tianhe-2 Caps Top 10 Supercomputers. IEEE Spectrum. Retrieved from http://spectrum.ieee.org/tech-talk/computing/hardware/tianhe2-caps-top-10-supercomputers

Attig, Norbert. JUQUEEN-Jülich's New Green Flagship. (2012, August 28). Retrieved from http://www.fz-juelich.de/SharedDocs/Meldungen/IAS/JSC/EN/2012/2012-04-juqueen.html

Balfour, J., Dally, W., Black-Schaffer, D., Parikh, V., \& Park, J. (2008). An Energy-Efficient Processor Architecture for Embedded Systems. Computer Architecture Letters, 7, 29-32. https://doi.org/10.1109/L-CA.2008.1

CEA-HPC. TGCC Curie. (2012, August 28). Retrieved from http://www-hpc.cea.fr/en/complexe/tgcc-curie.htm

Eisymont, L. K., \& Butov, A. A. (2016, September 14). Dynamically reconfigurable DRCP computing device (Unpublished presentation). 14 slides.

Eisymont, L. K., \& Nikitin, A. I. (2016, March 11). Cryptoraptor, P-VLSI, macroblocks (Unpublished presentation). 31 slides.

Eisymont, L. K. (2016, April 28). About the introduction of SFU slaves with static reconfiguration into a macroblock SVP (Unpublished presentation). 23 slides.

Khazraee, M., Gutierrez, L. V., Magaki, I., \& Tailor, M. B. (2017, May/June). Specializing a Plan-et's Computation: ASIC Clouds. IEEE Micro, 62-69. https://doi.org/10.1109/MM.2017.49

Li, W., Zeng, X. Y., Nan, L. M., Chen, T., \& Dai, Z. B. (2016, January). A reconfigurable Block Cryptographic Processor Based on VLIW Architecture. China Communications, 91-99. https://doi.org/10.1109/CC.2016.7405707

Li, W., Zeng, X. Y., Dai, Z. B., Nan, L. M., Chen, T., \& Ma, C. (2017, November). A High Energy-Efficient Reconfigurable VLIW Symmetric Cryptographic Processor with Loop Buffer Structure and Chain Processing Mechanism. Chainese Journal of Electronics, 26, 1161-1167. https://doi.org/10.1049/cje.2017.06.010

Molyakov, A. S., \& Eisymont, L. K. (2017). Technological Methods Analysis in the Field of Exaflops Supercomputers Devel-opment Approaching. Global Journal of Computer Science and Technology: Information \& Technology, 17, 37-44. Retrieved from https://globaljournals.org/GJCST_Volume17/6-Technological-Methods-Analysis-.pdf

\section{Copyrights}

Copyright for this article is retained by the author(s), with first publication rights granted to the journal.

This is an open-access article distributed under the terms and conditions of the Creative Commons Attribution license (http://creativecommons.org/licenses/by/4.0/). 